\title{
Comparative clinical study of efficacy and safety of adapalene $0.1 \%$ gel versus benzoyl peroxide $2.5 \%$ gel for the treatment of acne vulgaris
}

\author{
Anuradha Dubey ${ }^{1}$, Hanmant Amane ${ }^{2}$
}

\author{
${ }^{1}$ Department of Pharmacology, \\ L.N. Medical College and \\ Research Centre, Bhopal, \\ Madhya Pradesh, India \\ ${ }^{2}$ Department of Pharmacology, \\ G.M.E.R.S. Medical College, \\ Junagadh, Gujarat, India
}

Received: 16 August 2019

Accepted: 13 September 2019

*Correspondence to:

Dr. Anuradha Dubey,

Email: dranudubey999@ gmail.com

Copyright: (C) the author(s), publisher and licensee Medip Academy. This is an openaccess article distributed under the terms of the Creative Commons Attribution NonCommercial License, which permits unrestricted noncommercial use, distribution, and reproduction in any medium, provided the original work is properly cited.

\begin{abstract}
Background: Acne vulgaris is characterised by comedones, papules, pustules and nodules occurring in a sebaceous distribution. Topical treatments, such as adapalene and benzoyl peroxide, are popular in mild to moderate acne vulgaris. This study was aimed to compare the efficacy and safety of adapalene with benzoyl peroxide in the patients of mild to moderate acne vulgaris.

Methods: We planned a randomized, open-labelled, prospective study to compare the efficacy and side effects of adapalene and benzoyl peroxide in acne patients. A total of 100 patients with mild to moderate acne vulgaris were included in the study. They were randomly divided into 2 groups with 50 patients in each group. One group was given $0.1 \%$ adapalene gel and the other group received $2.5 \%$ benzoyl peroxide gel. Efficacy was assessed as reduction in the lesion counts, whereas for safety, the side effects like dryness, burning, irritation, erythema were recorded during the treatment. Total duration of the study was 3 months.

Results: The study revealed that adapalene was more effective than benzoyl peroxide in treating non-inflammatory and inflammatory lesions of acne vulgaris, and there was a statistically significant difference found between the groups $(\mathrm{p} \leq 0.05)$ in efficacy. Adapalene was also found to be comparatively safer than benzoyl peroxide because the patients treated with adapalene had lesser side effects than those treated with benzoyl peroxide.

Conclusions: Our study concludes a better efficacy and safety of adapalene than benzoyl peroxide in the treatment of mild to moderate acne vulgaris.
\end{abstract}

Keywords: Acne, Lesion counts, Retinoids, Topical treatment

\section{INTRODUCTION}

Acne vulgaris is an inflammatory disorder which commonly affects the sebaceous glands on face, chest and back. ${ }^{1}$ It can be termed a chronic disease because of its long duration and recurring nature. ${ }^{2}$ Because it affects the appearance, the patients of acne may suffer from sad emotional state, this is also a reason why its treatment is very demanding. The pathogenesis of acne is a complex process which comprises abnormal lipid metabolism, dyskeratosis and bacterial growth. ${ }^{1}$ Topical treatments such as adapalene and benzoyl peroxide are popular in mid to moderate acne vulgaris. ${ }^{3}$
Adapalene is a third generation topical retinoid which is a synthetic naphthoic acid derivative with better efficacy and lesser side effects. It reverses the abnormal follicular desquamation and also has anti-inflammatory effects. ${ }^{4}$ Studies have also revealed that the topical retinoid act efficaciously against microcomedo, the natural precursor of comedones. 5

Benzoyl peroxide is another safe and effective topical agent for treating acne vulgaris. It has both anticomedogenic and antimicrobial properties, with no fear of antibiotic resistance. ${ }^{6}$ The main side effects of benzoyl peroxide are concentration-dependent skin 
irritation and dryness which can be prevented through using lower but still effective doses. ${ }^{7}$ Some studies have claimed that the efficacy of adapalene is comparable to that of benzoyl peroxide on non-inflammatory and inflammatory lesions of mild to moderate acne vulgaris. ${ }^{8,9}$ The aim of the present study was to evaluate and compare the efficacy and safety of adapalene and benzoyl peroxide in treatment of mild to moderate acne vulgaris.

\section{METHODS}

This study was a randomized, single centre, observational, comparative study in which 100 patients with mild to moderate acne vulgaris were recruited from a tertiary care hospital, from November 2012 to April 2014. The patients were recruited after approval from the institutional ethics committee and informed written consents were obtained from the participants before entering the study.

Male and female patients in the age group of 12 to 30 years with grade 2 and 3 of acne vulgaris (as per Investigator's Global Assessment of acne scale) were recruited. $^{2}$

The patients with severe acne or other dermatological conditions requiring systemic therapy, as well as pregnant or nursing women were not included in the study.

The patients were randomly divided into two groups with 50 patients in each group. Adapalene $0.1 \%$ gel was given to Group A patients whereas Group B patients got benzoyl peroxide $2.5 \%$ gel. They were instructed to apply a thin layer of gel, once daily at bed time on the whole affected area. The treatment duration was planned as 3 months and control visits were arranged with 1-month intervals.

For evaluation of efficacy, the 'lesion counting technique' was used in order to compare the effectiveness of treatment. The lesions were divided into noninflammatory lesions (open and closed comedones) and inflammatory lesions (papules, pustules, nodules, cyst). The lesions were counted before the therapy, during the control visits and after the treatment.

For safety evaluation, the adverse events like dryness, pain, burning, irritation, pruritus were reported throughout the study. All participants were evaluated for other possible side effects by interviewing and a complete physical examination at each visit.

\section{Statistical analysis}

For both the treatment groups, the descriptive statistics (mean and standard deviation) were calculated for noninflammatory, inflammatory and total lesion counts at each visit. One way analysis of variance was used to analyse the changes in the lesion counts in both the groups. The unpaired student t-test was used for comparing the lesion counts in between the groups. $\mathrm{p} \leq 0.05$ was considered statistically significant. No statistical analysis was performed for safety assessment, was only summarized by graphical representation.

\section{RESULTS}

Out of the total 100 patients enrolled, only 92 patients completed the treatment (47 patients in group A, 45 patients in group B). Eight patients were excluded because of noncompliance with the treatment regimen or the follow-up schedule. Out of 92 patients, 35 patients were males and 57 were females. Further classifying the patients on the basis of grade of acne, 27 patients were grade 2 and 65 patients were grade $3 .^{2}$ For both the treatment groups, a progressive and significant decline was observed in the number of non-inflammatory, inflammatory and total lesion counts throughout the study period.

In Group A, the mean count of non-inflammatory lesions was 25.91 at the first visit (baseline), which was reduced to $16.78,9.7$ and 3.95 at the end of one, two and three months respectively. The mean count of inflammatory lesions was 16.25 at the first visit, which was reduced to $10.25,5.37$ and 2 at the end of one, two and three months respectively. Whereas the mean count of total lesions was 28.68 during the first visit, which was reduced to 18.5 , 10.68 and 4.29 at the end of one, two and three months respectively (Table 1). Reduction of lesions before and after treatment with adapalene can be seen in Figure 1.

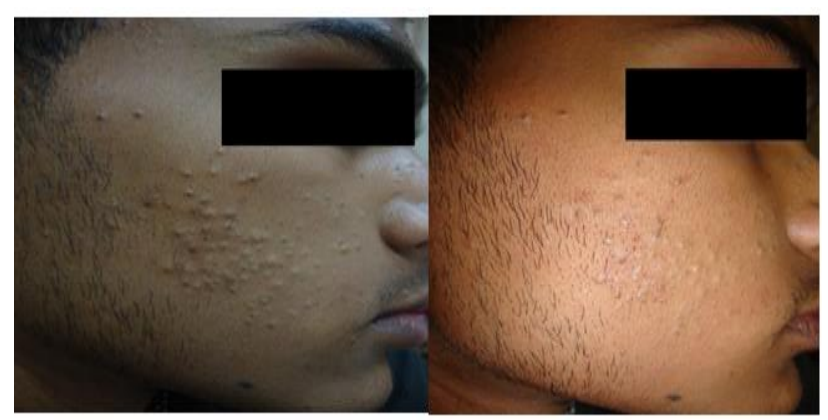

Figure 1: Reduction of lesions before and after treatment with adapalene.

In Group B, it was found that the mean count of noninflammatory lesions was 24.82 at the first visit (baseline), which was reduced to $17.93,12.08$ and 7.54 at the end of one, two and three months respectively. The mean count of inflammatory lesions was 16 at the first visit, which was reduced to $9,4.5$ and 3 at the end of one, two and three months respectively. Whereas the mean count of total lesions was 26.22 at the first visit, which was reduced to $18.71,12.47$ and 7.80 at the end of one, two and three months respectively (Table 2). 
Table 1: Reduction of lesions in adapalene group (Group A).

\begin{tabular}{|lllll|} 
Visits & $\begin{array}{l}\text { Visit 1 (baseline) } \\
(\text { mean } \pm \text { SD })\end{array}$ & Visit 2 $($ mean \pm SD $)$ & Visit 3 (mean \pm SD) & Visit 4 (mean \pm SD $)$ \\
\hline NIL & $25.91 \pm 10.4$ & $16.78 \pm 8.0$ & $9.7 \pm 6.44$ & $3.95 \pm 4.0$ \\
\hline IL & $16.25 \pm 3.99$ & $10.25 \pm 3.30$ & $5.37 \pm 2.19$ & $2 \pm 0.53$ \\
\hline Total lesions & $28.68 \pm 14.67$ & $18.5 \pm 10.77$ & $10.68 \pm 7.5$ & $4.29 \pm 4.55$ \\
\hline
\end{tabular}

$\mathrm{NIL}=$ Non-inflammatory lesions, $\mathrm{IL}=$ Inflammatory lesions, Visit-1= Baseline values, Visit-2= At the end of 1 month, Visit-3= At the end of 2 months, Visit-4= At the end of 3 months.

Table 2: Reduction of lesions in benzoyl peroxide group (Group B).

\begin{tabular}{|lllll|}
\hline Visits & $\begin{array}{l}\text { Visit } \mathbf{1}(\text { baseline }) \\
(\text { mean } \pm \text { SD })\end{array}$ & Visit 2 $($ mean \pm SD $)$ & Visit 3 (mean \pm SD $)$ & Visit 4 (mean \pm SD $)$ \\
\hline NIL & $24.82 \pm 10.83$ & $17.93 \pm 8.5$ & $12.08 \pm 7.14$ & $7.54 \pm 5.62$ \\
\hline IL & $16 \pm 3.74$ & $9 \pm 4.76$ & $4.5 \pm 2.51$ & $3 \pm 0.81$ \\
\hline Total lesions & $26.22 \pm 13.47$ & $18.71 \pm 9.87$ & $12.47 \pm 7.75$ & $7.80 \pm 6.08$ \\
\hline
\end{tabular}

Table 3: Comparison of non-inflammatory lesions before and after the treatment.

\begin{tabular}{|lllll|}
\hline \multirow{2}{*}{ Lesions } & \multicolumn{2}{l|}{ Adapalene group (A) } & \multicolumn{2}{l|}{ Benzoyl peroxide group (B) } \\
\cline { 2 - 3 } & Baseline & After 3 months & Baseline & After 3 months \\
\hline NIL & 1218 & 186 & 1142 & 347 \\
\hline
\end{tabular}

Baseline $=0$ month, After 3 months=end of therapy.

Table 4: Comparison of inflammatory lesions before and after the treatment.

\begin{tabular}{|lllll|}
\hline \multirow{2}{*}{ Lesions } & \multicolumn{2}{l|}{ Adapalene group (A) } & \multicolumn{2}{l|}{ Benzoyl peroxide group (B) } \\
\hline IL & Baseline & After 3 months & Baseline & After 3 months \\
\hline
\end{tabular}

Baseline $=0$ month, After 3 months=end of therapy.

Table 5: Comparison of total lesions before and after the treatment.

\begin{tabular}{|lllll|}
\hline \multirow{2}{*}{ Lesions } & \multicolumn{2}{l|}{ Adapalene group (A) } & \multicolumn{2}{l|}{ Benzoyl peroxide group (B) } \\
\hline Total & Baseline & After 3 months & Baseline & After 3 months \\
\hline
\end{tabular}

Baseline $=0$ month, After 3 months=end of therapy.

Table 6: Percentage of reduction in non-inflammatory lesions.

\begin{tabular}{|lllll|}
\hline Visits & Visit-1 (baseline) & Visit-2 & Visit-3 & Visit-4 \\
\hline Group A & 0 & $37.25 \pm 11.63$ & $65.35 \pm 15.66$ & $87 \pm 12.33$ \\
\hline Group B & 0 & $28.45 \pm 9.36$ & $53.79 \pm 14.58$ & $72.34 \pm 15.87$ \\
\hline
\end{tabular}

Reduction of non-inflammatory lesion, inflammatory lesion and total lesion counts from baseline values were highly significant in both the groups $(\mathrm{p}<0.0001)$.

In non-inflammatory lesions, in both the groups, there was statistically significant difference present in different visits $(\mathrm{p}<0.0001)$, and between the groups also, there was a significant difference in different visits $(\mathrm{p}<0.05)$ (Table $6)$.

Likewise in inflammatory lesions, in both the groups, there was statistically significant difference present in different visits $(\mathrm{p}<0.0001)$, and between the groups also, there was a significant difference in different visits $(\mathrm{p}<0.05)$ (Table 7).

Similarly, in total lesion reduction, in both the groups, there was statistically significant difference present in different visits $(\mathrm{p}<0.0001)$, and between the groups also, there was significant difference in different visits $(\mathrm{p}<0.05)$ (Table 8). These findings indicate better efficacy of adapalene as compared to benzoyl peroxide for the treatment of mild to moderate acne vulgaris. 
Table 7: Percentage of reduction in inflammatory lesions.

\begin{tabular}{|lllll|}
\hline Visits & Visit-1 (baseline) & Visit-2 & Visit-3 & Visit-4 \\
\hline Group A & 0 & $37.53 \pm 8.05$ & $67.23 \pm 7.45$ & $87.6 \pm 1.02$ \\
\hline Group B & 0 & $47.18 \pm 23.52$ & $73.81 \pm 11.63$ & $81.35 \pm 1.0$ \\
\hline
\end{tabular}

Table 8: Percentage of reduction in total lesions

\begin{tabular}{|lllll|}
\hline Visits & Visit-1 (baseline) & Visit-2 & Visit-3 & Visit-4 \\
\hline Group A & 0 & $37.70 \pm 10.98$ & $66.13 \pm 14.85$ & $87.9 \pm 10.7$ \\
\hline Group B & 0 & $28.86 \pm 9.25$ & $54.43 \pm 14.25$ & $72.8 \pm 15.41$ \\
\hline
\end{tabular}

According to our study, adapalene also showed a better safety profile than benzoyl peroxide, there was only the problem of dryness which was seen in $2.1 \%$ patients treated with adapalene, whereas among the patients, who had applied benzoyl peroxide, 6.5\% suffered from burning and another $6.5 \%$ had irritation. Adverse events did not interfere with completion of treatment in the patients (Figure 2).

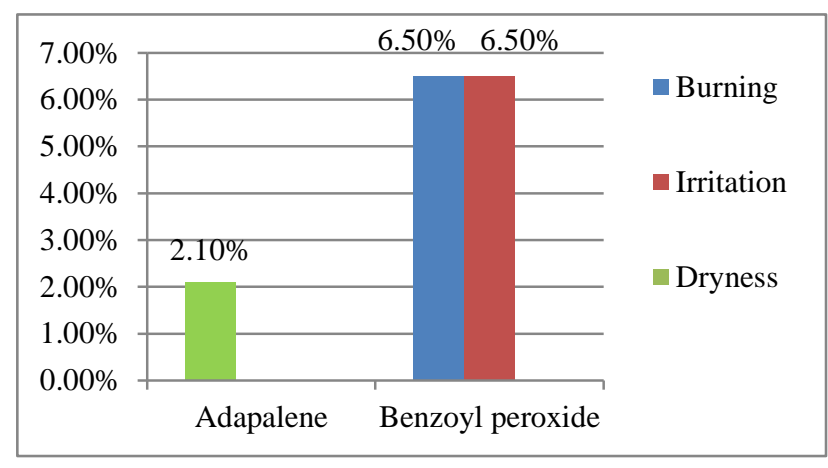

Figure 2: Adverse drug reactions.

\section{DISCUSSION}

The literally meaning of the term acne is "point, edge" which means a "skin eruption". Acne develops as a result of seborrhea, comedo formation, colonization of the intrafollicular duct with Propionibacterium acnes. Acne vulgaris is characterized by comedones, papules, pustules, nodules in a sebaceous distribution (face, upper chest, back). ${ }^{2}$

For mild to moderate acne vulgaris, topical therapy is the standard treatment. ${ }^{10}$ The retinoids and benzoyl peroxide are frequently used drugs for the topical treatment. Benzoyl peroxide is a bactericidal agent that has moderate comedolytic and anti-inflammatory action. ${ }^{10}$ Topical retinoids reduce the abnormal growth and development of keratinocytes within pilosebaceous duct. ${ }^{11}$ Adapalene, which is a topical retinoid, binds to specific retinoic acid nuclear receptors, and modulates the cellular differentiation, keratinization and inflammatory processes. $^{12}$

Despite the fact that there are a lot of studies with benzoyl peroxide, or adapalene alone, there are only a few studies which compare these two drugs. ${ }^{7,13-15}$ Therefore our aim was to compare adapalene and benzoyl peroxide in terms of their safety and efficacy in the topical treatment of acne vulgaris. In our study, reduction of non-inflammatory lesion, inflammatory lesion and total lesion counts from baseline values were highly significant in both the groups $(\mathrm{p}<0.0001)$, and between the groups also, there was a statistically significant difference present in different visits $(p<0.05)$. These findings conclude the better efficacy of adapalene as compared to benzoyl peroxide for the treatment of mild to moderate acne vulgaris. This data correlates with the study conducted by Babaeinejad and Fouladi, they found adapalene to be significantly more superior to benzoyl peroxide in reducing the lesions of mild acne vulgaris. ${ }^{3}$ But they found a faster onset of action of benzoyl peroxide (at month 1) against inflammatory lesions, which they concluded to be due to its more rapid and superficial antibacterial and anti-inflammatory functions. The difference between the groups for inflammatory lesions returned back to insignificant levels from the month 2 onwards. Like our study, this study was also a single centre, randomized study comparing efficacy and safety of adapalene $0.1 \%$ gel and benzoyl peroxide $2.5 \%$ gel for a study period of 3 months, but they used only 60 patients with mild acne vulgaris. ${ }^{3}$

In a similar study, Nascimento et al also compared the efficacy and safety of adapalene $0.1 \%$ gel and benzoyl peroxide, but the concentration of benzoyl peroxide was different, they used $4 \%$ benzoyl peroxide, and unlike our study, they found benzoyl peroxide to be more efficacious than adapalene on non-inflammatory and inflammatory lesions at weeks 2 and $5 .^{8}$

Korkut et al compared these two drug monotherapies with their combination, and concluded that the combination therapy has no superior efficacy over adapalene or benzoyl peroxide monotherapy. This was also an open-label, prospective study but unlike our study, they used $5 \%$ benzoyl peroxide lotion. ${ }^{9}$

The adverse events are also major determinants in selecting a topical medication for acne patients. In our study, we found lesser side effects with adapalene group than those with benzoyl peroxide. Only $2.1 \%$ patients treated with adapalene complained with the problem of 
dryness, whereas among the patients who were treated with benzoyl peroxide, $6.5 \%$ had burning and other $6.5 \%$ patients suffered with the problem of irritation. The adverse events did not interfere with the completion of the treatment, whereas in a study conducted by Babaeinejad and Fouladi, mild and transient side effects were found in both adapalene and benzoyl peroxide groups. $^{3}$ The patients treated with adapalene suffered from $13 \%$ dryness, $10 \%$ peeling, $3.3 \%$ redness whereas those treated with benzoyl peroxide had $10 \%$ dryness, $3.3 \%$ peeling and $3.3 \%$ burning. $^{3}$

It has been justified that benzoyl peroxide $2.5 \%$ is as effective as higher concentrations (5\% and 10\%) in treating acne vulgaris while causing fewer side effects. ${ }^{7}$ In the study conducted by Nascimento et al, both adapalene and benzoyl peroxide were found to be safe drugs although they used $4 \%$ benzoyl peroxide. ${ }^{8}$

Similarly, Korkut et al also concluded that there were no significant differences between the 3 groups (adapalene, benzoyl peroxide and adapalene-benzoyl peroxide combination) with regard to erythema, dryness or burning. All the side effects diminished with the continuation of the treatment. ${ }^{9}$

\section{CONCLUSION}

Adapalene has got better efficacy and safety than benzoyl peroxide, thus adapalene monotherapy can be used for the treatment of mild to moderate acne vulgaris whereas severe inflammatory lesions of acne can be topically treated with combining adapalene with other drugs, along with the systemic therapy as suggested by previous studies.

\section{ACKNOWLEDGEMENTS}

We are thankful to the faculty and staff of department of Dermatology for their support in this study.

Funding: No funding sources

Conflict of interest: None declared

Ethical approval: The study was approved by the Institutional Ethics Committee

\section{REFERENCES}

1. Kawashima M, Nagare T, Masaharu DOI. Clinical efficacy and safety of benzoyl peroxide for acne vulgaris: comparison between Japanese and Western patients. J Dermatol. 2017;44:1-7.

2. Gowda A, Sanmitha SK, Satish KS. Comparative study of clinical efficacy and side effects of adapalene $0.1 \%$ gel and benzoyl peroxide $2.5 \%$ gel as monotherapies and combination therapy in facial acne: Indian prospective. J Evol Med Dent Sci. 2014;3:3786-98.
3. Babaeinejad SH, Fouladi RF. The efficacy, safety and tolerability of adapalene versus benzoyl peroxide in the treatment of mild acne vulgaris; a Randomized trial. J Drug Dermatol. 2013;12:1033-8.

4. Czernielewski J, Michel S, Bouclier M, Baker M, Hensby JC. Adapalene biochemistry and the evolution of a new topical retinoid for treatment of acne. J Eur Acad Dermatol Venereol. 2001;15:5-12.

5. Thielitz A, Sidou F, Gollnick H. Control of microcomedone formation throughout a maintainance treatment with adapalene gel, 0.1\%. J Eur Acad Dermatol Venereol. 2007;21:747-53.

6. Gupta AK, Lynde CW, Kunynetz RA, Amin S, Choi K, Goldstein E. A randomized, double-blind, multicentre, parallel group study to compare relative efficacies of the topical gels 3\% erythromycin/ $5 \%$ benzoyl peroxide and $0.025 \%$ tretinoin/erythromycin $4 \%$ in the treatment of moderate acne vulgaris of the face. J Cutan Med Surg. 2003;7:31-7.

7. Mills $\mathrm{OH}$, Kligman AM, Pochi P, Comite $\mathrm{H}$. Comparing $2.5 \%, 5 \%$, and $10 \%$ benzoyl peroxide on inflammatory acne vulgaris. Int $\mathbf{J}$ Dermatol. 1986;25:664-7.

8. do Nascimento LV, Guedes AC, Magalhaes GM, de Faria FA, Guerra RM, de C Almeida F. Single-blind and comparative clinical study of the efficacy and safety of benzoyl peroxide $4 \%$ gel (BD) and adapalene $0.1 \%$ gel (QID) in the treatment of acne vulgaris for 11 weeks. J Dermatol Treat. 2003;14:166-71.

9. Korkut C, Piskin S. Benzoyl peroxide, adapalene and their combination in the treatment of acne vulgaris. $\mathrm{J}$ Dermatol. 2005;32:169-73.

10. Rathi SK. Acne vulgaris treatment: the current scenario. Indian J Dermatol. 2011;56:7-13.

11. Christophers E, Wolfe HH. Effect of Vitamin A acid on skin; in vitro and in vivo studies. Acta Derm Venereol (Stockh). 1975;74:42-53.

12. Shalita A, Weiss J, Chalker D, Ellis C, Greenspan A, $\mathrm{Katz} \mathrm{H}$, et al. A comparison of the efficacy and safety of adapalene gel $0.1 \%$ and tretinoin gel $0.025 \%$ in the treatment of acne vulgaris: a multicentre trial. J Am Acad Dermatol. 1996;34:482-5.

13. Smith EB, Padilla RS, McCabe JM, Becker LE. Benzoyl peroxide lotion (20\%) in acne. Cutis. 1980;25:90-2.

14. Lucky A, Jorizzo JL, Rodriguez D, Jones TM, Stewart DM, Tschen EH. Efficacy and tolerance of adapalene cream $0.1 \%$ compared with its cream vehicle for the treatment of acne vulgaris. Cutis. 2001;68:34-40.

15. Baker M, Tuley M, Busdiecker FL, Herndon JH, Slayton RM. Adapalene gel $0.1 \%$ is effective and well tolerated in acne patients in a dermatology practice setting. Cutis. 2001;68:41-7.

Cite this article as: Dubey $\mathrm{A}$, Amane $\mathrm{H}$.

Comparative clinical study of efficacy and safety of adapalene $0.1 \%$ gel versus benzoyl peroxide $2.5 \%$ gel for the treatment of acne vulgaris. Int J Basic Clin Pharmacol 2019;8:2317-21. 